\title{
Bie Jia Jian Pill Combined with Bone Mesenchymal Stem Cells Regulates microRNA-140 to Suppress Hepatocellular Carcinoma Stem Cells
}

\author{
Huang Jingjing ${ }^{1,2, *}$, Huang Hongna ${ }^{3, *}$, Zhang Wenfu ${ }^{4}$, Lv Jianlin ${ }^{4}$, Huang Guochu ${ }^{1}$, Lin Yuanjia ${ }^{1}$, \\ Chen Songlin ${ }^{5}$, Hu Yueqiang ${ }^{3,6}$ \\ ${ }^{1}$ Department of Spleen, Stomach and Liver Diseases, The First Affiliated Hospital of Guangxi University of Chinese Medicine, \\ Nanning, China \\ ${ }^{2}$ Guangxi Key Laboratory of Translational Medicine for Treating High-Incidence Infectious Diseases with Integrative Medicine, \\ Nanning, China \\ ${ }^{3}$ Teaching and Research Office of Internal Medicine of Traditional Chinese Medicine, The First Affiliated Hospital of Guangxi \\ University of Chinese Medicine, Nanning, China \\ ${ }^{4}$ Department of Internal Medicine, The First Affiliated Hospital of Guangxi University of Chinese Medicine, Nanning, China \\ ${ }^{5}$ Department of Human Resources, The First Affiliated Hospital of Guangxi University of Chinese Medicine, Nanning, China \\ ${ }^{6}$ Guangxi Key Laboratory of Basic Research of Traditional Chinese Medicine, Nanning, China
}

Background and Objectives: Cancer stem cells (CSCs) with tumorigenic potential are reported as the crucial factors of hepatocellular carcinoma (HCC) recurrence and therapy resistance. Bone mesenchymal stem cells (BMSCs) are documented to play an important role in the protection of hepatocytes. Bie Jia Jian pill (BJJP), a Traditional Chinese Medicine, has been used to treat liver fibrosis and liver cancer. This study aimed to explore the potential role of combined use of BJJP with BMSCs in HCC cell lines.

Methods and Results: Flow cytometry was used to identify BMSCs isolated from BALB/c mice and CSCs enriched from Huh7 cells by measuring CD24, CD133, CD44, CD73, CD105, CD166, CD29, CD14 and CD34. Differentiation potential of BMSCs was also determined. Cell viability and proliferation ability of CSCs were determined by CCK- 8 assay and clone formation assay. The expressions of CSCs biomarkers and Wnt/ $\beta$-catenin signal pathway related proteins were determined by PCR and western blot. TOP-Flash/FOP-Flash luciferase assay was applied to measure the activity of $\beta$-catenin/TCF. Compared with untreated CSCs, BJJP or BMSCs treatment alone on CSCs lead to increased miR-140 expression and cell apoptosis, as well as decreased expressions of CD24, CD133, EpCAM and cell viability. Downregualted expressions of Wnt/ $\beta$-catenin signal pathway related proteins, Wnt3a and $\beta$-catenin were found in response to BJJP or BMSCs treatment alone. The combination of BJJP+BMSCs treatment on CSCs could further enhance the suppressive effect on CSCs. Down-regulation of miR-140 in CSCs partially blocked the effects of BMSCs or BMSCs+BJJP on the expressions of Wnt3a and $\beta$-catenin as well as the cell viability and apoptosis of CSCs. Reversed expression pattern was found in CSCs transfected with miR-140 overexpression.

Conclusions: Taken together, we demonstrate that BJJP+BMSCs together could further enhance the suppressive effect on CSCs through regulating miR-140 and suppressing Wnt/ $\beta$-catenin signal pathway. This study demonstrated the potential of BJJP+BMSCs in therapeutic treatment of HCC.

Keywords: Hepatocellular carcinoma, Cancer stem cells, Bie Jia Jian pill, Bone mesenchymal stem cells, microRNA 140

Received: September 28, 2020, Revised: December 10, 2020, Accepted: January 20, 2021, Published online: February 28, 2021

Correspondence to $\mathrm{Hu}$ Yueqiang

Teaching and Research Office of Internal Medicine of Traditional Chinese Medicine, The First Affiliated Hospital of Guangxi University of Chinese Medicine, No. 89-9, Dongge Road, Qingxiu District, Nanning 530200, China

Tel: +86-13152510678, E-mail: huyq@gxtcmu.edu.cn

*These authors contributed equally to this work.

(c) This is an open-access article distributed under the terms of the Creative Commons Attribution Non-Commercial License (http://creativecommons.org/licenses/by-nc/4.0/), which permits unrestricted non-commercial use, distribution, and reproduction in any medium, provided the original work is properly cited.

Copyright (c) 2021 by the Korean Society for Stem Cell Research 


\section{Introduction}

Hepatocellular carcinoma (HCC), a major form of liver cancer, ranks the second leading cause of cancer related death globally (1). Nowadays, surgical resection combined with radiotherapy, chemotherapy and biotherapy have maintained the mainstream for HCC therapy strategy (2). However, the majority of HCC patients are diagnosed with advanced liver cancer due to an asymptomatic early stage, which results in the unavoidable systemic metastasis when surgery option is no longer available (3). Additionally, resistances to radiotherapy and chemotherapy always occur in HCC patients (4).

Cancer stem cells (CSCs) are the specific subpopulations of cells with stem cell-like characteristics in tumor tissues (5). CSCs have the potentials of self-renewal and differentiation, which may be responsible for the recurrence and metastasis of HCC (6). Bone mesenchymal stem cells (BMSCs) are cells derived from pluripotent bone marrow, characterized by easy obtaining and culture, and vigorous proliferation ability (7). Emerging evidence demonstrate the implication of BMSCs in the development and progression of various cancers via interacting with tumor cells $(8,9)$, but how BMSCs works in tumor microenvironment remains much to be determined.

MicroRNAs (miRNAs) belong to the small non-coding RNAs with 20 25 nucleotides in length. MiRNAs can degrade or inhibit the translation of the targeted gene by binding to the 3' untranslated region (UTR) of mRNA in various conditions (10). Among which, microRNA 140 (miR-140) has been documented as a tumor suppressor in HCC (11-13). The association of miR-140 with BMSCs was proved in chondrocyte cells (14), but whether this association still stands in HCC remains unclear.

Traditional Chinese Medicine (TCM) has been used in the adjuvant treatment of HCC with satisfactory therapeutic efficiency $(15,16)$. Bie Jia Jian pill (BJJP) is a kind of TCM from "the Synopsis of Prescription of the Golden Chamber". BJJP has been used for the therapy of liver fibrosis or hepatitis B cirrhosis (17). A previous studies highlighted the importance of BJJP on suppression HCC progression, whose mechanism involves MAPK signal pathway (18). According to collected data, $80 \sim 90 \%$ of HCC patient develops from liver fibrosis or cirrhosis (19). However, the effects of BJJP on HCC are unknown. In this present study, BJJP and BMSCs were used to treat CSCs enriched from Huh7 cells to investigate their effects on CSCs. This study identified that both BJJP and BMSCs can suppress the CSCs viability. In addition, BJJP and BMSCs together could further exert their protective effect against CSCs through regulating miR-140 and $\mathrm{Wnt} / \beta$ catenin signal pathway.

\section{Materials and Methods}

\section{Animals}

A total of 26 male BALB/c mice (18 $\pm 2 \mathrm{~g})$ provided by the Hubei Provincial Laboratory Animal Public Service Center were housed in the specific pathogen free (SPF) animal room under the $12 \mathrm{~h} \mathrm{light/dark}$ cycles at $24 \sim 26^{\circ} \mathrm{C}$ with free access to food and water. All experiments were conducted in accordance with the Guidelines for the Institutional Animal Care and Use Committee (IACUC) and approved by the Committee of The First Affiliated Hospital of Guangxi University of Chinese Medicine.

\section{Enrichment of stem cell-like Huh7 spheres}

Human HCC cells (Huh7) were purchased from iCell (Shanghai, China). First, Huh7 cells were cultured in high glucose DMEM medium (iCell, Shanghai, China) supplemented with $10 \%$ fetal bovine serum $(\mathrm{FBS})$ at $37^{\circ} \mathrm{C}$ in a humidified incubator with $5 \%(\mathrm{v} / \mathrm{v}) \mathrm{CO}_{2}$. After digestion and centrifugation, Huh7 cells were resuspended with stem cell conditioned culture medium [low glucose DMEMF12 medium containing B27 (1: 50), 0.4\% BSA, EGF (20 $\mathrm{ng} / \mathrm{ml})$ and $\mathrm{bFGF}(20 \mathrm{ng} / \mathrm{ml})]$. Then, the cells were plated into 24 well ultra-low attachment plates with serum-free stem cell conditioned culture medium to form the spheres. The spheres were collected after 8 days. Sphere-like cells were trypsinized into single cell suspension for follow-up experiments.

\section{Isolation and culture of BMSCs}

Male $\mathrm{BALB} / \mathrm{c}$ mice $(\mathrm{n}=6)$ were sacrificed by cervical dislocation. Then mice were then soaked in $75 \%(\mathrm{v} / \mathrm{v})$ ethanol for $10 \mathrm{~min}$. Under aseptic condition, the femur and tibia were removed. After the epiphysis at both ends was removed, BMSCs in the bone marrow cavity were washed out with low-glucose DMEM. The culture medium containing cell suspension was centrifuged at low speed for $5 \mathrm{~min}$ and the supernatant was discarded, and then resuspended with low-glucose DMEM complete culture medium. The collected cells were cultured at $37^{\circ} \mathrm{C}$ in a humidified incubator with $5 \%(\mathrm{v} / \mathrm{v}) \mathrm{CO}_{2}$. After incubation for $48 \mathrm{~h}$, the non-adherent cells were removed. When confluence was $80 \% \sim 90 \%$, the adherent cells were harvested by trypsin digestion and designated BMSCs.

\section{Cell transection}

miR-140 overexpression cells were established by trans- 
fecting miR-140 mimic into CSCs using the lipofectamine 3,000 reagent (Invitrogen, USA) according to the manufacturer's instructions. MiR-140 knockdown cells were established by transfecting miR-140 inhibitor into CSCs using the lipofectamine 3,000 reagent (Invitrogen, USA) according to the manufacturer's instructions. Sequences for miRNAs transfection were shown as follows: miR-140 mimic NC: TTCTCCGAACGTGTCACGT; miR-140 mimic: CAGTGGTTTTACCCTATGGTAG; miR-140 inhibitor NC: CAGTACTTTTGTGTAGTACAA; miR-140 inhibitor: CTACCATAGGGTAAAACCACTG.

\section{Preparation of drug-containing serum}

Male BALB/c mice $(n=20)$ received intragastric administration of saline $(0.9 \%)$ once a day for continuous 15 days. The other $10 \mathrm{BALB} / \mathrm{c}$ mice received intragastric administration of BJJP (1.1 g/ kg, Sinopharm Zhonglian, Wuhan, China) once a day for continuous 15 days. After the mice were anesthetized with pentobarbital sodium, the blood was collected from the carotid artery and centrifuged at $4^{\circ} \mathrm{C}$ for $10 \mathrm{~min}(3,000 \mathrm{r} / \mathrm{min})$. The obtained serum was inactivated at $56^{\circ} \mathrm{C}$ for 20 minutes, filtered and sterilized at $0.22 \mu \mathrm{m}$, and stored at $4^{\circ} \mathrm{C}$.

\section{Cell culture and treatments}

Using the transwell chamber as the co-culture system, BMSCs and CSCs were directly co-cultured in a nontouch manner. Briefly, CSCs with different treatments were inoculated in the lower chamber with or without serum containing BJJP. BMSCs were inoculated in the upper chamber. After cultured in a humidified incubator with $5 \%(\mathrm{v} / \mathrm{v}) \mathrm{CO}_{2}$ at $37^{\circ} \mathrm{C}$ for 7 days, CSCs in the lower chamber were used for the subsequent experiments.

\section{Measurement on surface antigen}

Flow cytometry analysis was conducted to evaluate the cell surface marker expressions of the CSCs. CSCs with various treatments were harvested and trypsinized into a single cell suspension. Subsequently, cells were incubated with fluorochrome-conjugated antibodies, including FITCconjugated anti-human cluster of differentiation (CD) 24 (eBioscience, CA, USA), APC-conjugated anti-human CD133 (Biolegend, CA, USA) at $4{ }^{\circ} \mathrm{C}$ in the dark. The appropriated isotype-matched antibodies were used as negative controls. After $30 \mathrm{~min}$ incubation, the samples were centrifuged and washed with PBS for flow cytometry analysis. CSCs with positive expressions of both CD24 and CD133 were selected for further experiments. Similarly, the cell surface marker expressions of the BMSCs were also evaluated by flow cytometry. The conjugated-specific anti- bodies used were as follows: PE-conjugated anti-mouse CD14 (Biolegend, CA, USA), APC-conjugated anti-mouse CD34 (Biolegend, CA, USA), APC-conjugated anti-mouse CD45 (Biolegend, CA, USA), PE-conjugated anti-mouse CD44 (Biolegend, CA, USA), PE-conjugated anti-mouse CD73 (Biolegend, CA, USA), APC-conjugated anti-mouse CD105 (Biolegend, CA, USA), FITC-conjugated antimouse CD166 (abcam, CA, USA), FITC-conjugated antimouse CD29 (Biolegend, CA, USA). Cell analysis was performed using a Flow cytometer (BD Biosciences, CA, USA) and FolwJo software version 7.6 (FlowJo LLC, OR, USA).

\section{Differentiation ability of BMSCs}

Adipogenic differentiation by oil red $\mathrm{O}$ staining: BMSCs $\left(2 \times 10^{4}\right.$ cells $\left./ \mathrm{cm}^{2}\right)$ were inoculated into the 6 well plates for cell fusion. Adipogenic differentiation was induced with adipogenic differentiation basal medium (MUCMX90031, Cyagen, Guangzhou, China) when cell fusion reaches $100 \%$. BMSCs were added with $2 \mathrm{ml}$ of culture medium for OriCell BALB/C BMSCs culture medium (liquid A) after the original culture medium was removed. After adipogenic differentiation for 3 day, the liquid $\mathrm{A}$ in the 6 well plates was removed and replaced with $2 \mathrm{ml}$ of culture medium for OriCell BALB/C BMSCs culture medium (liquid B) for cell culture for $24 \mathrm{~h}$. Then the liquid $\mathrm{B}$ was absorbed and replaced with liquid $\mathrm{A}$. The inter-replacement of liquid A and liquid B was performed for 3 5 times (12 20 days), the BMSCs were cultured with liquid $B$ for $4 \sim 7$ day (refresh every $2 \sim 3$ days) until the lipid droplets are big enough. The 6 well plates were washed with $1 \times$ PBS for 1 2 times and fixed with $2 \mathrm{ml} \mathrm{4 \%} \mathrm{neu-}$ tral formalin solution per well for $30 \mathrm{~min}$. Then the formalin solution was removed and the 6 well plates were washed with $1 \times$ PBS for twice. After PBS wash, each well was added with $1 \mathrm{ml}$ of oil red $\mathrm{O}$ dye liquor for $30 \mathrm{~min}$ (dye liquor formulate: oil red $\mathrm{O}:$ distill water $=3: 2$, filtered through the neutral filter paper). After the oil red $O$ dye liquor was removed, the 6 well plates were washed in $1 \times$ PBS for $2 \sim 3$ times and observed under a microscope.

Osteogenic differentiation and alizarin red staining: BMSCs of $2 \times 10^{4}$ cell $/ \mathrm{cm}^{2}$ were seeded into the gelatin coated 6 well plates. Osteogenic differentiation was induced with Osteogenic differentiation culture medium (MUCMX-90031, Cyagen, Guangzhou, China) when cell fusion reaches $60 \sim 70 \%$. After the culture medium was removed, the 6 well plates were added with culture medium for $2 \mathrm{ml}$ OriCell BALB/C BMSCs (refresh every 3 days) for $2 \sim 4$ weeks. Alizarin red was used for cell staining. 
After staining, the culture medium was removed and the 6 well plates were washed with $1 \times$ PBS for $1 \sim 2$ times, followed by fixation with $2 \mathrm{ml} \mathrm{4 \%} \mathrm{neutral} \mathrm{formalin} \mathrm{solution}$ per well for $30 \mathrm{~min}$. Then the formalin solution was removed and the 6 well plates were washed with $1 \times \mathrm{PBS}$ for twice. After PBS wash, each well was added with $1 \mathrm{ml}$ of alizarin red. About 30 min later, the 6 well plates was washed with $1 \times \mathrm{PBS}$ for $2 \sim 3$ times and then observed under a microscope.

\section{CCK8 assay}

Cell counting kit-8 (CCK-8) assay was conducted to evaluate the cell viability of CSCs. A total of $10 \mu 1$ of CCK-8 reagent (Beyotime, Shanghai, China) was added into each well and then incubated for another $2 \mathrm{~h}$. The absorbance of each well at $450 \mathrm{~nm}$ was assessed using a microplate reader (Diatek, Wuxi, China).

\section{Clone formation assay}

Cells in logarithmic phase were digested with $0.25 \%$ trypsin and made into single cells for cell suspension in culture medium containing $10 \%$ fetal calf serum. Cell suspensions were seed into pre-warmed culture disk which contains $10 \mathrm{ml}$ of culture medium at the density of 50 , 100 and 200 cells per disk. Cells were gently shaken and then maintained at $37^{\circ} \mathrm{C}$ environment with $5 \%$ saturated humidity for 2 to 3 weeks. Culture was terminated once cell clones were visible by naked eyes. The cell suspension was removed and the cells were washed in PBS for twice and fixed in $5 \mathrm{ml}$ of acetic acid/methanol (1:3) for 15 min. After that, cell clones were stained with Giemsa staining for 10 to $30 \mathrm{~min}$ and washed in running water. The air dryed culture disks were inversed and counted for cell clones. Cell clones with more than 10 cells were counted to calculate the cell clone rate.

\section{Cell apoptosis assay}

Cell apoptosis of CSCs was determined using Annexin $\mathrm{V}$ cell apoptosis kit (Sungene Biotech, Tianjin, China) following the manufacturer's protocol. The harvested CSCs were washed with PBS and resuspended in the Annexin $\mathrm{V}$ binding buffer. Subsequently, cells were incubated with $5 \mu 1$ of Annexin V-PE and $5 \mu 1$ of 7-AAD solution at room temperature for $15 \mathrm{~min}$ in the dark and analyzed by flow cytometry (Becton Dickinson Co., CA, USA).

\section{Fluorescent quantitative polymerase chain reaction}

Total RNA was extracted from the harvested CSCs using TRIpure Total RNA Extraction Reagent (ELK Biotechnology, Wuhan, China) following the manufacturer's protocol. Reverse-transcription was conducted using EntiLink $^{\mathrm{TM}}$ 1st Strand cDNA Synthesis Kit (ELK Biotechnology, Wuhan, China). Next, the synthesized cDNA was amplified using the EnTurbo ${ }^{\mathrm{TM}}$ SYBR Green PCR SuperMix Kit (ELK Biotechnology, Wuhan, China). Relative expression levels of the targeted gene were calculated using the $2^{-\Delta \Delta \mathrm{Ct}}$ method. For CD24, CD133 and epithelial cell adhesion molecule (EpCAM), GAPDH served as the internal control. For miR-140, U6 served as the internal control. The sequences of PCR primers are listed in Table 1.

\section{Western blot}

The harvested CSCs were lysed with RIPA Lysis Buffer (Aspen, Canada, USA) containing the protease inhibitor cocktail (Roche, Basel, Switzerland), followed by centrifugation at $12000 \mathrm{rpm}$ at $4{ }^{\circ} \mathrm{C}$ for $5 \mathrm{~min}$. Protein concentration was determined by BCA protein kit (Aspen, Canada, USA). Total protein was electrophoresed on $10 \%$ SDS-PAGE and then transferred to PVDF membrane. Subsequently, the membrane was incubated with the primary antibodies overnight at $4^{\circ} \mathrm{C}$, followed by the corresponding horseradish peroxidase conjugated secondary antibodies at room temperature for $30 \mathrm{~min}$. GAPDH served as the internal control. Protein bands were captured by enhanced chemiluminescence (ECL) substrate solution kit (Aspen, Canada, USA) for $1 \mathrm{~min}$ and analyzed with AlphaEaseFC software. Primary antibodies used in this study are listed in Table 2.

\section{TOP-Flash/FOP-Flash luciferase assay}

Cells in logarithmic phase were seeded in 24-well plates at the density of $1 \times 10^{5} / \mathrm{ml}$ for cell culture for $24 \mathrm{~h}$. The

Table 1. The primer sequences used in this study

\begin{tabular}{cll}
\hline Gene & & \multicolumn{1}{c}{ Sequence $\left(5^{\prime}-3^{\prime}\right)$} \\
\hline CD24 & Forward & ACCCACGCAGATTTATTCCAG \\
& Reverse & CACGAAGAGACTGGCTGTTGAC \\
CD133 & Forward & GCACTCTATACCAAAGCGTCAAG \\
& Reverse & GCACGATGCCACTTTCTCAC \\
EpCAM & Forward & GTGTGTGAACACTGCTGGGGT \\
& Reverse & CTGAAGTGCAGTCCGCAAACT \\
miR-140 & Forward & GGGTAGAACCACGGCTCAAC \\
& Reverse & CTCAACTGGTGTCGTGGAGTC \\
GAPDH & Forward & CATCATCCCTGCCTCTACTGG \\
& Reverse & GTGGGTGTCGCTGTTGAAGTC \\
U6 & Forward & CTCGCTTCGGCAGCACAT \\
& Reverse & AACGCTTCACGAATTGCGT \\
\hline
\end{tabular}

CD24: cluster of differentiation 24, CD133: cluster of differentiation 133, EpCAM: epithelial cell adhesion molecule. 
Table 2. The primary antibodies used for western blot analyses in this study

\begin{tabular}{|c|c|c|c|c|}
\hline Antibody & Species & Manufacturer & Cat. No & Dilution ratio \\
\hline GAPDH & Rabbit & Abcam & ab9485 & $1: 10,000$ \\
\hline $\mathrm{CD} 24$ & Rabbit & Abcam & ab179821 & $1: 500$ \\
\hline CD133 & Rabbit & Cell Signaling Technology & 64326 & $1: 500$ \\
\hline ЕрСАM & Rabbit & Abcam & ab223582 & $1: 1,000$ \\
\hline Wnt3a & Rabbit & Abcam & ab219412 & $1: 500$ \\
\hline$\beta$-catenin & Rabbit & Abcam & ab32572 & $1: 500$ \\
\hline
\end{tabular}

CD24: cluster of differentiation 24, CD133: cluster of differentiation 133, EpCAM: epithelial cell adhesion molecule.

A

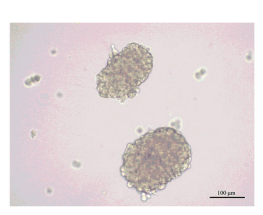

B

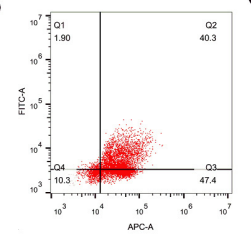

C

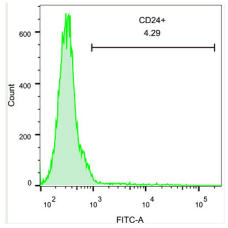

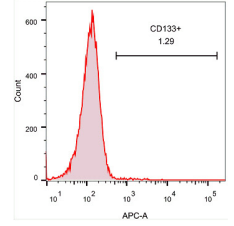

D
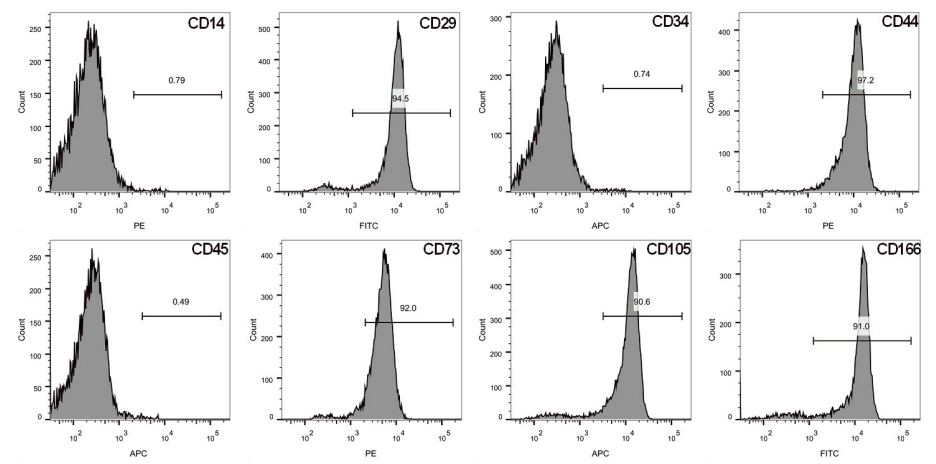

E

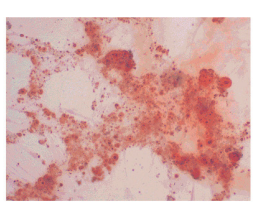

$\mathbf{F}$

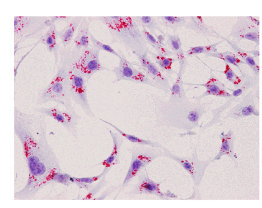

Fig. 1. Identification of isolated CSCs and BMSCs and their potential of osteogenic differentiation and adipogenic differentiation ability. (A) CSCS sphere; $(\mathrm{B}, \mathrm{C})$ measurement on CSCs biomarker, CD24 and CD133 by Flow cytometry in spheres and in Huh 7 cells; (D) surface antigens of BMSCs, CD44, CD73, CD105, CD166, CD29, CD14, CD34 and CD45 were measured by Flow cytometry; (E) alizarin red staining for determination of osteogenic differentiation; (F) oil red $\mathrm{O}$ staining for determination of adipogenic differentiation. CSCs, cancer stem cells; BMSCs, bone mesenchymal stem cells; BJJP, Bie Jia Jian pill. mixture of TOP/FOP Flash and pRL-TK plasmid $(50: 1)$ was transfected into cells for cell culture of $48 \mathrm{~h}$ before cell lysis at room temperature of $15 \mathrm{~min}$. The FLuc and RLuc solution was prepared based on the instruction of kit (GeneCopoeia, Guangzhou, China). The luciferase activity was measured at the ultra-sensitive tubular chemiluminescence apparatus (Berthold, Stuttgart, Germany). The ratio of TOP Flash/FOP Flash should be calculated to reflect the luciferase activity.

\section{Statistical analysis}

All experiments were repeated three times and all data were presented as mean \pm standard deviation (SD). Comparisons between multiple groups were analyzed by Oneway ANOVA analysis using the GraphPad Prism 7.00 soft- ware (GraphPad Software, Inc., CA, USA). Data between two groups were analyzed using Student's $t$ test. $\mathrm{p}<0.05$ was considered statistically significant.

\section{Results}

\section{Identification of CSCs and BMSCs}

Huh7 cells were cultured with stem cell conditioned medium and formed large spheres after 8 days (Fig. 1A). Flow cytometry showed that both spheres and Huh7 cells had positive expression of specific stem cell markers CD24 and CD133. The expression rate of CD24 and CD133 in spheres was $40.3 \%$ (Fig. 1B), while that in Huh7 cells was less than 5\% (Fig. 1C). Above results suggest the successful isolation of CSCs. Flow cytometry 
showed that the isolated BMSCs were positive for surface antigens CD44, CD73, CD105, CD166 and CD29, while negative for antigens CD14, CD34 and CD45, indicating the successful isolation of BMSCs (Fig. 1D). Staining by oil red $\mathrm{O}$ staining and alizarin red staining in Fig. $1 \mathrm{E}$ and $1 \mathrm{~F}$ supported the potential of osteogenic differentiation and adipogenic differentiation ability of BMSCs. Collectively, isolated CSCs and BMSCs are qualified for further experiments.

\section{BJJP + BMSCs inhibits the viability of CSCs and promotes cell apoptosis}

Previous study supported the implication of BMSCs in tumor development $(8,20)$. Therefore in this study we further explore the possible effect of BJJP or/and BMSCs in CSCs. To this end, we used BJJP or BMSCs to treat CSCs before cell proliferation and apoptosis rate were calculated. The comparison showed that compared with control group, CSCs biomarker CD24, CD133 and EpCAM were suppressed (Fig. 2A and 2B, p<0.05) in BJJP and BMSCs groups. Meanwhile, we also found suppressed cell viability and proliferation (Fig. 2C and 2D, p $<0.05$ ) as well as increased cell apoptosis rate (Fig. $2 \mathrm{E}, \mathrm{p}<0.05$ ) in BJJP and BMSCs groups in contrast to control group. Collectively, both BJJP and BMSCs can suppress the viability and enhance cell apoptosis of CSCs.

To verify the possible interaction of BJJP and BMSCs in CSCs, CSCs were co-treatment with BMSCs and BJJP. The results showed that compared with BJJP group or BMSCs group, the CD24, CD133 and EpCAM were suppressed while cell viability and proliferation ability of CSCs were suppressed in BJJP+BMSCs group (Fig. 2A $\mathrm{D}, \mathrm{p}<0.05)$. We also found increased cell apoptosis rate in BJJP+BMSCs group when compared with BJJP group or BMSCs group (Fig. 2E, $\mathrm{p}<0.05$ ). Collectively, combination use of BJJP and BMSCs could further suppress the cell growth of CSCs and promote cell apoptosis.
A

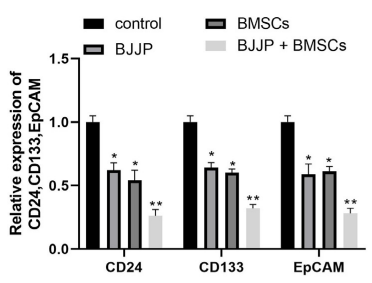

B

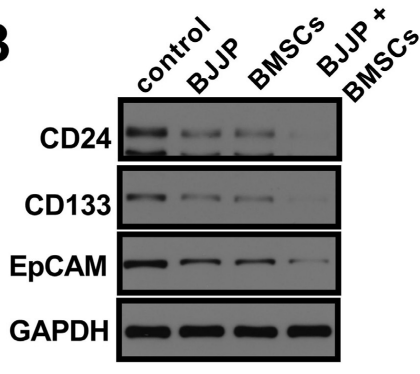

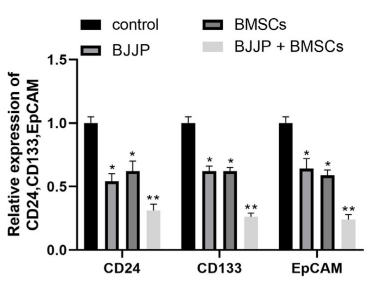

C
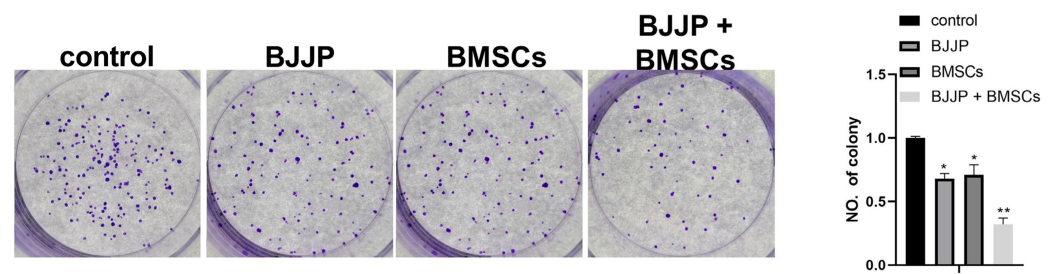

D

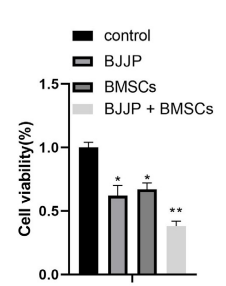

E
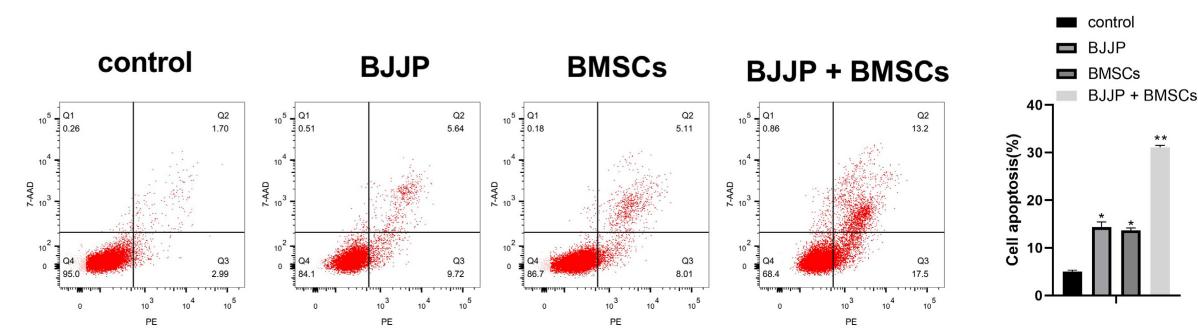

Fig. 2. Combination use of BJJP and BMSCs further suppress the cell growth of CSCs in addition to enhancing cell apoptosis. (A, B) the expressions of CSCs biomarkers CD24, CD133 and EpCAM were measured with RT-qPCR and western blot; (C) cell proliferation ability was assessed by cell clone formation assay; (D) cell viability was determined by CCK-8 assay; (E) cell apoptosis rate was assessed by Flow cytometry. $\mathrm{N}=3,{ }^{*} \mathrm{p}<0.05, * * \mathrm{p}<0.01$; EpCAM, epithelial cell adhesion molecule; CSCs, cancer stem cells; BMSCs, bone mesenchymal stem cells; BJJP, Bie Jia Jian pill. 


\section{BJJP and BMSCs promoted the expression of miR-140 in CSCs}

Based on previous research, miR-140 was believed to suppress the proliferation of CSCs with the potential of

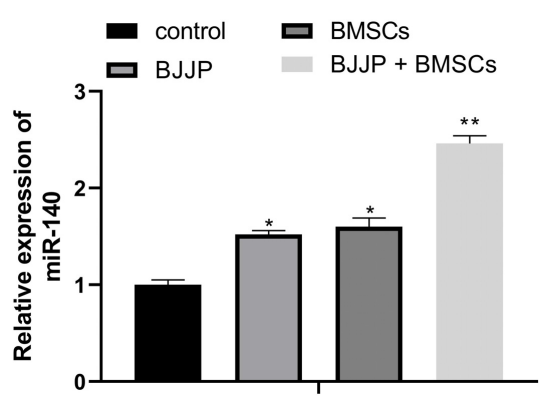

Fig. 3. miR-140 expression was increased in response to BJJP or/and BMSCs treatment as determined by RT-qPCR. $\mathrm{N}=3$, ${ }^{*} \mathrm{p}$ $<0.05, * * p<0.01$. BMSCs, bone mesenchymal stem cells; BJJP, Bie Jia Jian pill. serving as a tumor therapeutic target $(21,22)$. RT-qPCR demonstrated that compared with control group, the Huh7 cells had much higher expression level of miR-140 (Fig. $3, \mathrm{p}<0.05)$. We measured the expression of miR-140 in CSCs with or without BJJP/BMSCs treatment. The analysis on miR-140 expression showed that compared with control group, BJJP or BMSCs treatment alone could increase the expression of miR-140, while the expression of miR-140 was further elevated in BJJP+BMSCs group when compared with BJJP or BMSC group (Fig. 3, p $<0.05$ ). Collectively, both BJJP and BMSCs could upregulate miR-140 in CSCs.

\section{BJJP and BMSCs suppress CSCs through up-regulating miR-140}

miR-140 inhibitor was transfected into CSCs to verify whether miR-140 was involved in the suppressive effect of BJJP and BMSCs in CSCs. The transfection efficiency by
A
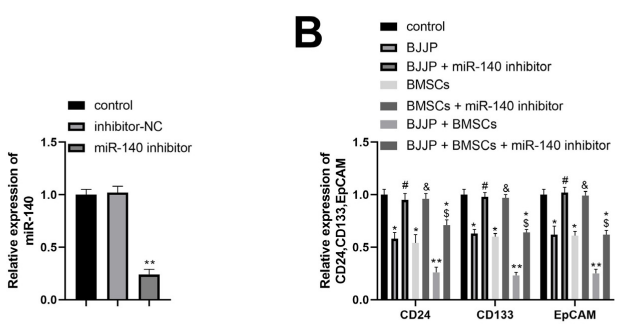

C

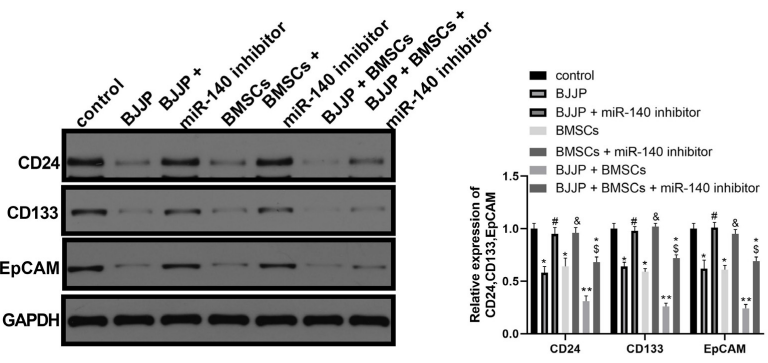

E

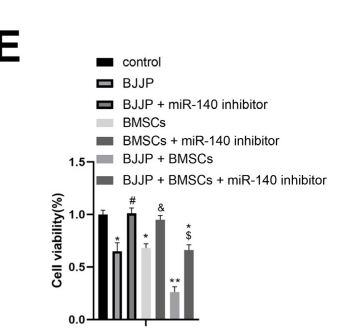

$\mathbf{F}$
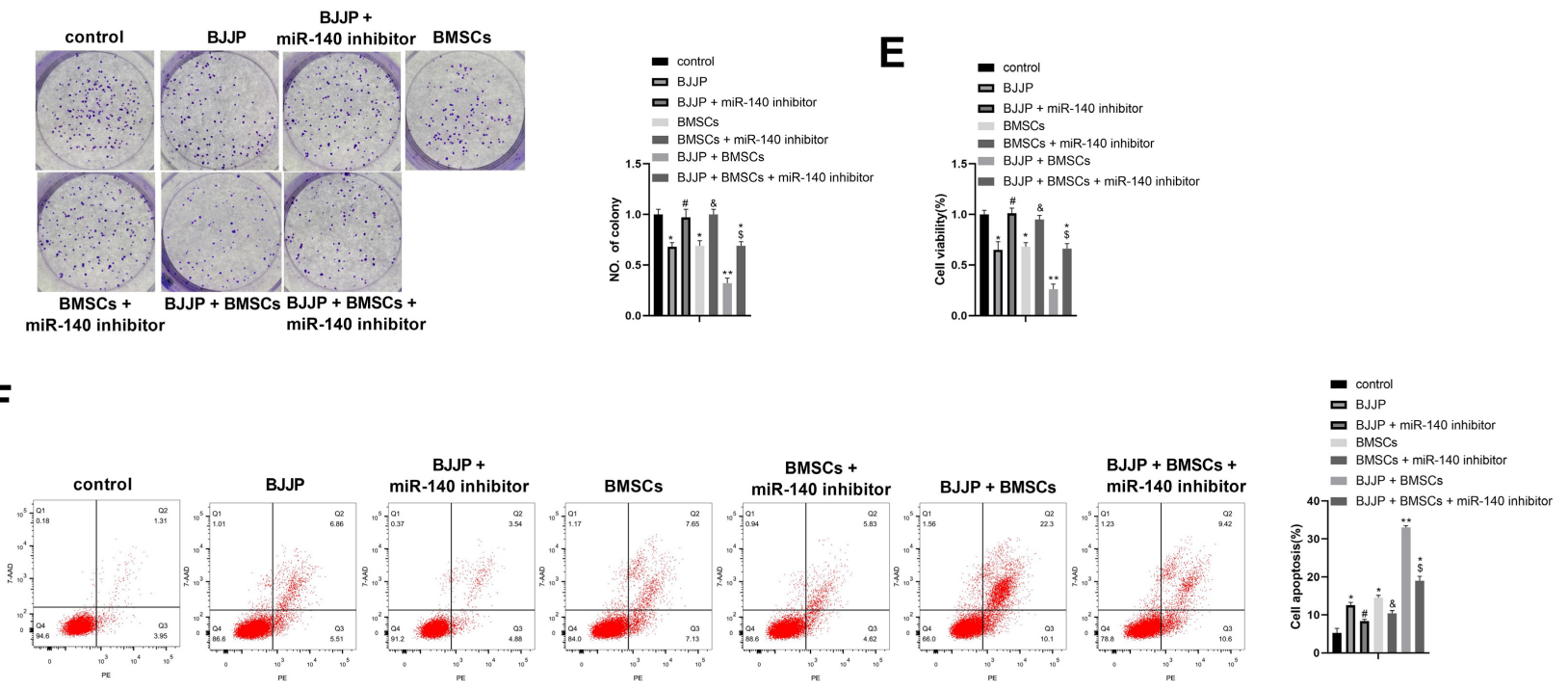

Fig. 4. BJJP and BMSCs suppress CSCs cell proliferation and enhance cell apoptosis through regulating miR-140. (A) Transfection efficiency was verified by RT-qPCR; (B, C) RT-qPCR and western blot were applied to detect the expression of CD24, CD133 and EpCAM after cell transfection; (D) cell proliferation ability was assessed by cell clone formation; (E) cell viability was measured by CCK-8 assay; (F)

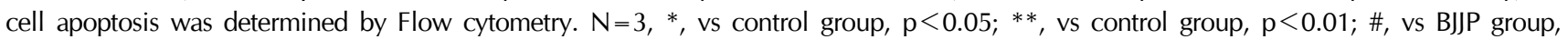
$\mathrm{p}<0.05$; *, vs BMSCs group, $\mathrm{p}<0.05$; *, vs BJJP+BMSCs group, $\mathrm{p}<0.05$; EpCAM, epithelial cell adhesion molecule; CSCs, cancer stem cells; BMSCs, bone mesenchymal stem cells; BJJP, Bie Jia Jian pill. 
A

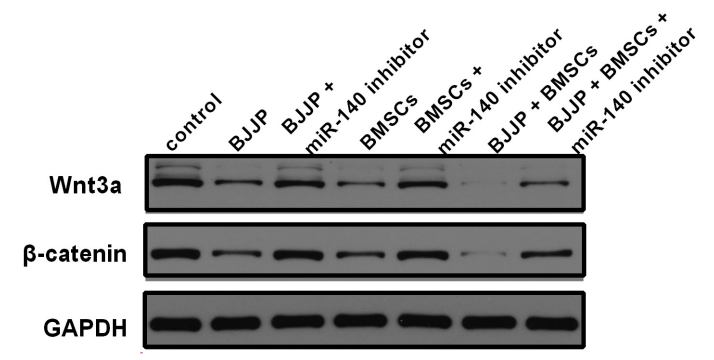

B

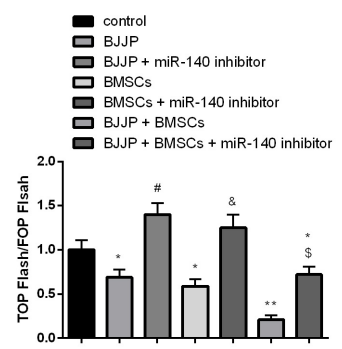

RT-qPCR showed that miR-140 was downregulated in miR-140 inhibitor group when compared with inhibitor-NC (Fig. 4A, p<0.05), suggesting the transfection efficiency was satisfactory. Detection on CSCs transfected with miR-140 inhibitor showed that inhibition on miR140 could partially abandon the suppressive effect of BJJP or BMSCs on cell growth of CSCs (Fig. $4 \mathrm{~B} \sim \mathrm{F}, \mathrm{p}<0.05$ ). Collectively, BJJP and BMSCs could up-regulating miR-140 expression to suppress the cell growth of CSCs.

\section{BJJP and BMSCs inhibits Wnt/ $\beta$-catenin signal pathway in CSCs}

Wnt/ $\beta$-catenin signal pathway plays a vital role in regeneration and renewal of CSCs $(23,24)$. Western blot was applied to detect the expressions of $\mathrm{Wnt} / \beta$-catenin signal pathway related proteins (Fig. 5A). In contrast to control group, BMSCs or BJJP treatment lead downregulated expressions of Wnt3a and $\beta$-catenin, meanwhile, BMSCs + BJJP group had further decreased Wnt3a and $\beta$-catenin expression when compared with BMSCs or BJJP treatment alone $(p<0.05)$. However, knockdown of miR-140 could rescue the expressions of Wnt3a and $\beta$-catenin $(\mathrm{p}<0.05)$. Luciferase assay in Fig. 5B on activity of $\beta$-catenin/TCF was consistent with that of $\beta$-catenin. Collectively, BJJP and BMSCs could upregulate miR-140 and suppress Wnt/ $\beta$-catenin signal pathway to suppress the cell growth of CSCs.

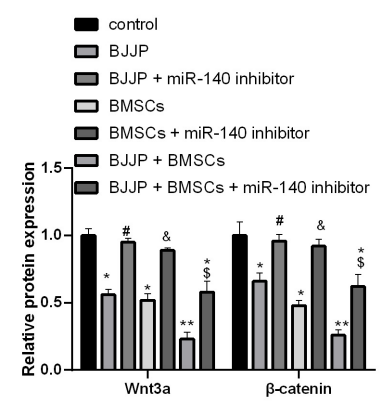

Fig. 5. BJJP and BMSCs could suppress the activation of $\mathrm{Wnt} / \beta$-catenin signal pathway through regulating miR-140. (A) Western blot was applied to measure the proteins related to $\mathrm{Wnt} / \beta$-catenin signal pathway. (B) The activity of $\beta$-catenin was measured by TOP-Flash/FOP-Flash luciferase assay. $\mathrm{N}=3, *$ vs control group, $\mathrm{p}<0.05 ;{ }^{* *}$ vs control group, $\mathrm{p}<0.01$; \# vs BJJP group, $\mathrm{p}<0.05$; * vs BMSCs group, $\mathrm{p}<0.05 ; *$, vs BJJP+BMSCs group, $\mathrm{p}<0.05$. BMSCs, bone mesenchymal stem cells; BJJP, Bie Jia Jian pill.

\section{Discussion}

HCC is a kind of cancer with high mortality and high recurrence rate due to the advanced diagnosis, drug resistance and other factors. In this study, we initially explored the possible role of BJJP in the progression of HCC. The collected evidence in this study showed that both BJJP and BMSCs can suppress the viability of CSCs and enhance cell apoptosis in HCC cell models. Furthermore, we also found the combination use of BJJP and BMSCs could enhance the suppressive effect on CSCs proliferation, among which the mechanism involves the upregulation of miR-140 and suppression of Wnt/ $\beta$-catenin signal pathway.

Mounting evidence showed that CSCs are associated with cancer recurrence, metastasis, therapy resistance and poor prognosis $(25,26)$. Lin et al. (27) have found that liver CSCs with tumorigenic potential shall be responsible for relapse and therapy resistance in HCC. CD24, CD133 and EpCAM are three markers of CSCs, which are usually related to the poor prognosis of various cancers (5). EpCAM, a hepatic CSCs biomarker, has been demonstrated to be implicated in poor clinical outcomes of HCC (28). In this study, the expressions of CD 24, CD133 and EpCAM were detected in CSCs enriched from Huh7 cells, which indicated that these cells possessed the potential of stem cells. We found that BJJP markedly reduced the levels of CD 24, CD133 and EpCAM in CSCs, suggesting the inhibitory effect of BMSCs on stem cell-like capacities. Moreover, the inhibitory effect of BJJP on cell viability 
and the promoting effect of BJJP on cell apoptosis were also demonstrated. Based on the obtained results, we suppose that BJJP inhibit the tumorigenic potential of CSCs and would be beneficial for the treatment of HCC.

BMSCs are cells with muti-differentiate potentials, which can differentiate into osteoblasts, nerve cells, chondrocytes and adipocytes in different conditions. BMSCs play important role in the resistance of liver fibrosis and the protection of liver cells (29). It has been documented that multipotent adult progenitor cells (MAPCs) from BMSCs could further differentiate into cells with the functional capacity of hepatocytes in vitro (30). In vitro studies have found that MSCs derived from fetal skin inhibit the growth of human liver cancer cells, manifested by the reduced cell proliferation, colony formation and oncogene expressions (31). As we previously reported, the combination of human MSCs and Rougan Huaqian granules attenuate the liver fibrosis associated with liver cancer (32). In this study, BMSCs were extracted from BALB/c mice. The identification of BMSCs cell phenotype was firstly conducted, evidenced by the positive expressions of CD29, CD44, CD73, CD105 and CD166, and the negative expressions of CD14, CD34 and CD45, which is consistent with the previous studies (33). These results confirmed the successful isolation of BMSCs form BALB/c mice. Subsequently, we co-cultured BMSCs with CSCs, and observed the effects of BMSCs on the functions of CSCs. We discovered that BMSCs significantly suppressed the expressions of three markers of CSCs (CD24, CD133 and EpCAM). Furthermore, BMSCs significantly inhibited the cell viability of CSCs and promoted the apoptosis of CSCs. These results demonstrate that BMSCs affect the functions of CSCs and may be beneficial for HCC treatment. Collected evidence in this study highlighted that the combined use of BJJP and BMSCs achieved better therapeutic effect on HCC progression. Data in a previous study exploring the effect of BJJP drug serum on facilitating the transformation of hepatocyte to BMSCs, therefore we speculated that BJJP may able to enhance the suppressive effect of BMSCs on HCC cells by enhancing the transformation of hepatocyte to BMSCs (34). Aberrant expression of miR-140 has been reported in liver cancer and demonstrated as a liver tumor suppressor (12). Yang et al. (11) have found that miR-140 inhibits the proliferation, invasion and metastasis of HCC cells by down-regulating TGF-bR1 and FGF9. Lu et al. (13) have reported the promoting role of the 1ncRNAFLJ33360/miRNA-140/MMP regulatory loop in the metastasis of HCC. In our study, we discovered that BMSCs alone, BJJP alone or BMSCs combined with BJJP treatment of CSCs markedly pro- moted the expression of the anti-tumor gene miR-140 in CSCs. Importantly, knockdown of miR-140 in CSCs increased the levels of three markers of CSCs, cell viability and reduced cell apoptosis of CSCs administrated with BMSCs or BMSCs combined with BJJP. These results confirm that miR-140 is a tumor suppressor gene in HCC and is beneficial for inhibiting the tumorigenic potential of CSCs. Additionally, BJJP and BMSCs inhibit the tumorigenic potential of CSCs through regulating miR-140. A study in esophagus cancer showed that miR-140 could suppress ZEB2 to regulate Wnt/ $\beta$-catenin signal pathway (35) and in melanoma, miR-140 was reported to target SOX4 so as to regulate $\mathrm{Wnt} / \beta$-catenin signal pathway (36). Further exploration on downstream target of miR-14 in CSCs showed that miR-140 knockdown could increase the expressions of Wnt3a and $\beta$-catenin. But our study did not further explore the mechanism of miR-140 regulation on Wnt/ $\beta$-catenin signal pathway. Western blot also showed that the combination use of BJJP with BMSCs could further suppress the expressions of Wnt/ $\beta$-catenin signal pathway related proteins. Taken together, BJJP and BMSCs may be able to suppress HCC progression via upregulating miR-140 to suppress the activation of $\mathrm{Wnt} / \beta$ -catenin signal pathway.

In summary, we demonstrated that BJJP combined with BMSCs exert satisfactory regulation on the functions of CSCs through regulating the expression of anti-tumor gene miR-140 and Wnt/ $\beta$-catenin signal pathway. There are several limitations of this study that should be addressed. Although our study highlighted the therapeutic effect of combination of BMSCs with BJJW on HCC, the explanation of how BJJP could have combination effect with BMSC was not mentioned in this study. Meanwhile, in vivo researches are required to investigate the potential mechanism of BJJP and BMSCs in HCC, which is the future direction for our study.

\section{Acknowledgments}

This study was supported by National Natural Science Foundation of China (Project no.: 81403382, 81760845) and by Promotive Research Fund for Excellent Young and Middle-aged teachers in Colleges and Universities of Guangxi Province (Project no.: 2019KY0313).

\section{Potential Conflict of Interest}

The authors have no conflicting financial interest.

\section{Author Contributions}

Huang Jingjing, Huang Hongna and $\mathrm{Hu}$ Yaoqiang contributed to the conception of the study; Hu Yaoqiang, 
Huang Jingjing and Huang Hongna performed the experiments; Huang Jingjing, Huang Hongna, Zhang Wenfu and Lv Jianlin contributed significantly to data analysis and manuscript preparation; Huang Guochu wrote the manuscript; Lin Yuanjia and Chen Songlin perform the analysis with constructive discussions; Hu Yaoqiang supervised and checked the manuscript.

\section{References}

1. Wu JZ, Jiang N, Lin JM, Liu X. STYXL1 promotes malignant progression of hepatocellular carcinoma via downregulating CELF2 through the PI3K/Akt pathway. Eur Rev Med Pharmacol Sci 2020;24:2977-2985

2. Ma W, Zhu D, Li J, Chen X, Xie W, Jiang X, Wu L, Wang G, Xiao Y, Liu Z, Wang F, Li A, Shao D, Dong W, Liu W, Yuan Y. Coating biomimetic nanoparticles with chimeric antigen receptor $\mathrm{T}$ cell-membrane provides high specificity for hepatocellular carcinoma photothermal therapy treatment. Theranostics 2020;10:1281-1295

3. El-Serag HB. Hepatocellular carcinoma. N Engl J Med 2011;365:1118-1127

4. Shao Y, Song X, Jiang W, Chen Y, Ning Z, Gu W, Jiang J. MicroRNA-621 acts as a tumor radiosensitizer by directly targeting SETDB1 in hepatocellular carcinoma. Mol Ther 2019;27:355-364

5. Padthaisong S, Thanee M, Namwat N, Phetcharaburanin J, Klanrit P, Khuntikeo N, Titapun A, Sungkhamanon S, Saya H, Loilome W. Overexpression of a panel of cancer stem cell markers enhances the predictive capability of the progression and recurrence in the early stage cholangiocarcinoma. J Transl Med 2020;18:64

6. Wu Y, Zhang J, Zhang X, Zhou H, Liu G, Li Q. Cancer stem cells: a potential breakthrough in HCC-targeted therapy. Front Pharmacol 2020;11:198

7. Rada T, Santos TC, Marques AP, Correlo VM, Frias AM, Castro AG, Neves NM, Gomes ME, Reis RL. Osteogenic differentiation of two distinct subpopulations of human adipose-derived stem cells: an in vitro and in vivo study. J Tissue Eng Regen Med 2012;6:1-11

8. Hong IS, Lee HY, Kang KS. Mesenchymal stem cells and cancer: friends or enemies? Mutat Res 2014;768:98-106

9. Yagi H, Kitagawa Y. The role of mesenchymal stem cells in cancer development. Front Genet 2013;4:261

10. Zhang Y, Shi K, Liu H, Chen W, Luo Y, Wei X, Wu Z. miR-4458 inhibits the epithelial-mesenchymal transition of hepatocellular carcinoma cells by suppressing the TGF- $\beta$ signaling pathway via targeting TGFBR1. Acta Biochim Biophys Sin (Shanghai) 2020;52:554-562

11. Yang H, Fang F, Chang R, Yang L. MicroRNA-140-5p suppresses tumor growth and metastasis by targeting transforming growth factor $\beta$ receptor 1 and fibroblast growth factor 9 in hepatocellular carcinoma. Hepatology 2013;58: $205-217$
12. Takata A, Otsuka M, Yoshikawa T, Kishikawa T, Hikiba Y, Obi S, Goto T, Kang YJ, Maeda S, Yoshida H, Omata M, Asahara H, Koike K. MicroRNA-140 acts as a liver tumor suppressor by controlling NF- $\kappa \mathrm{B}$ activity by directly targeting DNA methyltransferase 1 (Dnmtl) expression. Hepatology 2013;57:162-170

13. Lu Z, Yu Y, Ding X, Jin D, Wang G, Zhou Y, Zhu Y, Na L, He Y, Wang Q. LncRNA FLJ33360 accelerates the metastasis in hepatocellular carcinoma by targeting miRNA140/MMP9 axis. Am J Transl Res 2020;12:583-591

14. Mahboudi H, Soleimani M, Hanaee-Ahvaz H, Ghanbarian H, Bandehpour M, Enderami SE, Kazemi B. New approach for differentiation of bone marrow mesenchymal stem cells toward chondrocyte cells with overexpression of MicroRNA140. ASAIO J 2018;64:662-672

15. Liu X, Li M, Wang X, Dang Z, Yu L, Wang X, Jiang Y, Yang Z. Effects of adjuvant traditional Chinese medicine therapy on long-term survival in patients with hepatocellular carcinoma. Phytomedicine 2019;62:152930

16. Jianxin C, Qingxia X, Junhui W, Qinhong Z. A case of recurrent hepatocellular carcinoma acquiring complete remission of target lesion with treatment with traditional Chinese medicine. Integr Cancer Ther 2017;16:597-604

17. Zhang T, Yang Y, Wang B, Zheng X, Wang L, Feng X, Li G, Shi J, Cao N. Meta-analysis of influences of Biejiajian Pill combined with entecavir on serum liver fibrosis markers of compensatory period of hepatitis $b$ cirrhosis: protocol of systematic review and meta-analysis. Medicine (Baltimore) 2019;98:e18458

18. Chang XF, Song CH, Chen T, Chen HY, Han LM. Effect of BieJiaJianWan on human hepatocellular carcinoma cells in Bel-7402 through MAPK signal pathway. J Gannan Med Coll 2015;35:663-666

19. Affo S, Yu LX, Schwabe RF. The role of cancer-associated fibroblasts and fibrosis in liver cancer. Annu Rev Pathol 2017;12:153-186

20. Serakinci N, Tulay P, Kalkan R. Role of mesenchymal stem cells in cancer development and their use in cancer therapy. Adv Exp Med Biol 2018;1083:45-62

21. Yoshida A, Kitajima S, Li F, Cheng C, Takegami Y, Kohno S, Wan YS, Hayashi N, Muranaka H, Nishimoto Y, Nagatani N, Nishiuchi T, Thai TC, Suzuki S, Nakao S, Tanaka T, Hirose O, Barbie DA, Takahashi C. MicroRNA-140 mediates $\mathrm{RB}$ tumor suppressor function to control stem cell-like activity through interleukin-6. Oncotarget 2017;8:13872-13885

22. Wu D, Zhang J, Lu Y, Bo S, Li L, Wang L, Zhang Q, Mao J. miR-140-5p inhibits the proliferation and enhances the efficacy of doxorubicin to breast cancer stem cells by targeting Wnt1. Cancer Gene Ther 2019;26:74-82

23. Reya T, Clevers $\mathrm{H}$. Wnt signalling in stem cells and cancer. Nature 2005;434:843-850

24. Hou J, Zhao N, Zhu P, Chang J, Du Y, Shen W. Irradiated mesenchymal stem cells support stemness maintenance of hepatocellular carcinoma stem cells through Wnt/ $\beta$-catenin signaling pathway. Cell Biosci 2020;10:93

25. Liu D, Hong Y, Li Y, Hu C, Yip TC, Yu WK, Zhu Y, Fong 
CC, Wang W, Au SK, Wang S, Yang M. Targeted destruction of cancer stem cells using multifunctional magnetic nanoparticles that enable combined hyperthermia and chemotherapy. Theranostics 2020;10:1181-1196

26. Acikgoz E, Tatar C, Oktem G. Triptolide inhibits $\mathrm{CD}_{133^{+}}$/ $\mathrm{CD}_{4} 4^{+}$colon cancer stem cell growth and migration through triggering apoptosis and represses epithelial-mesenchymal transition via downregulating expressions of snail, slug, and twist. J Cell Biochem 2020;121:3313-3324

27. Lin Q, Wu Z, Yue X, Yu X, Wang Z, Song X, Xu L, He Y, Ge Y, Tan S, Wang T, Song H, Yuan D, Gong Y, Gao L, Liang X, Ma C. ZHX2 restricts hepatocellular carcinoma by suppressing stem cell-like traits through KDM2Amediated H3K36 demethylation. EBioMedicine 2020;53: 102676

28. Park DJ, Sung PS, Kim JH, Lee GW, Jang JW, Jung ES, Bae SH, Choi JY, Yoon SK. EpCAM-high liver cancer stem cells resist natural killer cell-mediated cytotoxicity by upregulating CEACAM1. J Immunother Cancer 2020;8:e000301

29. Wang ZC, Yang S, Huang JJ, Chen SL, Li QQ, Li Y. Effect of bone marrow mesenchymal stem cells on the Smad expression of hepatic fibrosis rats. Asian Pac J Trop Med 2014;7:321-324

30. Schwartz RE, Reyes M, Koodie L, Jiang Y, Blackstad M, Lund T, Lenvik T, Johnson S, Hu WS, Verfaillie CM. Multipotent adult progenitor cells from bone marrow differentiate into functional hepatocyte-like cells. J Clin Invest 2002;109:1291-1302
31. Khakoo AY, Pati S, Anderson SA, Reid W, Elshal MF, Rovira II, Nguyen AT, Malide D, Combs CA, Hall G, Zhang J, Raffeld M, Rogers TB, Stetler-Stevenson W, Frank JA, Reitz M, Finkel T. Human mesenchymal stem cells exert potent antitumorigenic effects in a model of Kaposi's sarcoma. J Exp Med 2006;203:1235-1247

32. Wang ZC, Yang S, Huang JJ, Chen SL, Li QQ Li Y. Effect of Rougan Huaqian granules combined with human mesenchymal stem cell transplantation on liver fibrosis in cirrhosis rats. Asian Pac J Trop Med 2014;7:576-581

33. Shi S, Zhang M, Guo R, Miao Y, Zhang M, Hu J, Xi Y, Li B. Feasibility of lentiviral-mediated sodium iodide symporter gene delivery for the efficient monitoring of bone marrow-derived mesenchymal stem cell transplantation and survival. Int J Mol Med 2014;34:1547-1554

34. Wen ZX, Huang JJ, Wang ZC. Research on the transformation of hepatocyte from bone marrow mesenchymal stem cells by Biejiajianwan drug serum. Guiding J Tradit Chin Med Pharm 2019;25:35-38

35. Yang S, Li X, Shen W, Hu H, Li C, Han G. MicroRNA-140 represses esophageal cancer progression via targeting ZEB2 to regulate Wnt/ $\beta$-catenin pathway. J Surg Res 2021;257: 267-277

36. Zhao G, Yin Y, Zhao B. miR-140-5p is negatively correlated with proliferation, invasion, and tumorigenesis in malignant melanoma by targeting SOX4 via the Wnt/ $\beta$-catenin and NF- $\kappa$ B cascades. J Cell Physiol 2020;235:2161-2170 